\title{
Endoscopic sphincteroplasty: a novel and safe alternative to papillotomy in the management of bile duct stones
}

\author{
P Mac Mathuna, P White, E Clarke, J Lennon, J Crowe
}

\begin{abstract}
Removal of bile duct stones during endoscopic retrograde cholangiopancreatography (ERCP) usually includes papillotomy. Papillotomy is associated with occasional complications and in addition, the longterm sequelae of papillotomy in young patients having laparoscopic cholecystectomy remain unclear. As an alternative to papillotomy, this study prospectively evaluated the efficacy and safety of endoscopic balloon sphincteroplasty to facilitate bile duct clearance. Of 32 patients with bile duct stones (diameter 3-30 mm) at ERCP, sphincteroplasty was considered inappropriate in four patients because of stone size (>20 mm) necessitating papillotomy for bile duct clearance. Spincteroplasty was performed in the remaining 28 patients to permit duct clearance by dormier basket, balloon or mechanical lithotripsy. The bile duct was cleared in 22 patients (79\%) while additional measures including papillotomy or stent insertion were required in the remaining six patients (21\%) because of stone size or technical difficulties. There was no associated papillary haemorrhage. Pancreatitis was seen in one patient (4\%) but resolved within 24 hours. Our preliminary experience suggests that sphincteroplasty is a safe and effective sphincter preservation technique that significantly reduces the necessity for papillotomy in the management of bile duct stones.
\end{abstract}

(Gut 1994; 35: 127-129)

Endoscopic retrograde cholangiopancreatography (ERCP) is the standard method for removal of bile duct stones. The advent of laparoscopic cholecystectomy will probably increase the demand for endoscopic bile duct clearance. ${ }^{12}$ ERCP usually includes papillotomy to facilitate stone extraction with the potential complications of haemorrhage, perforation, and pancreatitis. ${ }^{34}$ Apart from these immediate complications, legitimate concern has recently been expressed regarding the longterm sequelae of papillotomy for bile duct clearance in young patients having laparoscopic cholecystectomy. ${ }^{1}$ An effective endoscopic alternative to papillotomy incorporating sphincter preservation would be an advantage. Balloon dilatation is well established in the management of benign biliary strictures but papillary dilatation or 'sphincteroplasty' has received limited attention. ${ }^{5-7} \mathrm{We}$ report our preliminary experience of endoscopic balloon sphincteroplasty for the removal of bile duct stones.

\section{Patients and methods}

Thirty two consecutive patients with clinical and sonographic evidence of extrahepatic biliary obstruction had bile duct stones confirmed at ERCP. The mean age was 54 years with an age range of 25 to 84 including 26 female and six male patients. Seven patients had had cholecystectomy (four open and three laparoscopic). The mean number of bile duct stones was two (range 1-6) with a mean size of $8 \mathrm{~mm}$ (range 3 to $25 \mathrm{~mm}$ ) (Table). Coagulation indices were normal and each patient received conventional antibiotic prophylaxis. ERCP was performed under intravenous sedation with midazolam $5-10 \mathrm{mg}$ and pethidine $50-100 \mathrm{mg}$ with some patients also receiving hysocine $20-40 \mathrm{mg}$. Sphincteroplasty was considered inappropriate in four patients because of stone size $(>20 \mathrm{~mm})$ and a papillotomy was performed with successful bile duct clearance. Sphincteroplasty was carried out in the remaining 28 patients.

\section{SPHINCTEROPLASTY TECHNIQUE}

After diagnostic cholangiography at ERCP using an Olympus video duodenoscope with a $3 \cdot 2$ or $4 \cdot 2$ biopsy channel, a 0.21 guide wire (Axcess 21 , Wilson-Cook) is passed through the diagnostic cannula into the bile duct. A Maxforce 5 French balloon tipped biliary catheter (Microvasive, Boston, USA) with a total length of $180 \mathrm{~cm}$ and balloon length of $2 \mathrm{~cm}$ is then passed over the guide wire, positioning the deflated balloon across the papilla. The correct position of the balloon is confirmed in two ways, firstly by endoscopic visualisation and secondly by the position of two radio-opaque markers at each end of the balloon on $x$ ray screening. The balloon is

Clinical details, stone characteristics, outcome, and complications of patients having balloon sphincteroplasty

\begin{tabular}{ll}
\hline & Sphincteroplasty \\
\hline Patients & \\
Number & 28 \\
Mean age & 54 \\
Range & $26-84$ \\
Bile duct stones & 8 \\
Mean size $(\mathrm{mm})$ & $3-20$ \\
Size range $(\mathrm{mm})$ & 5 \\
Max number & $22 / 28(79 \%)$ \\
Efficacy & $2 / 28(7 \%)$ \\
Duct clearance & $6 / 28(21 \%)$ \\
Repeat ERCP & $4 / 28$ \\
Failed clearance & $2 / 28$ \\
Papillotomy & $1(4 \%)$ \\
Endoprosthesis & 0 \\
Complications & Pancreatitis \\
Haemorrhage & \\
\hline
\end{tabular}

$\mathrm{ERCP}=$ endoscopic retrograde cholangiopancreatography.
Gastrointestinal Unit Hospital, University Ireland 26 May 1993 


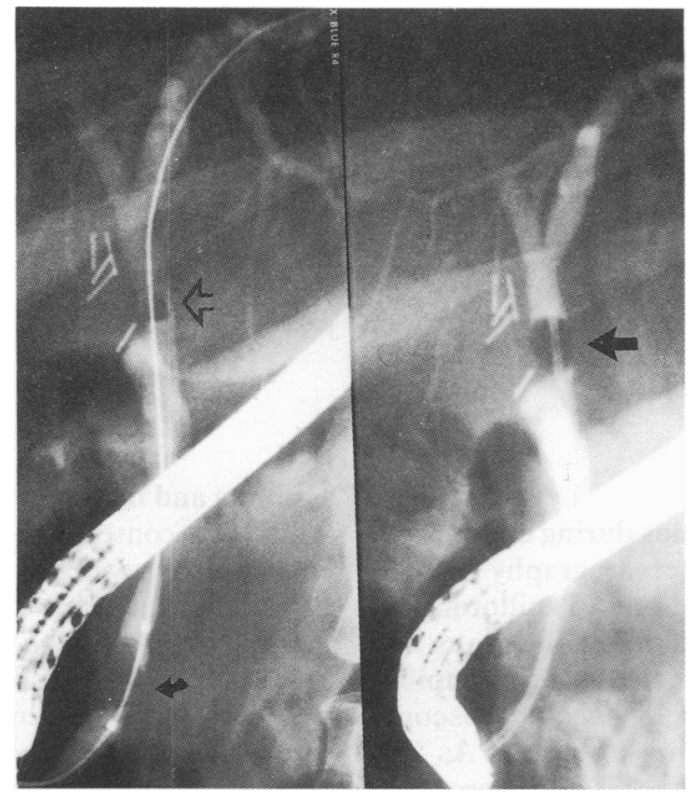

Left panel: sphincteroplasty balloon inflated across papilla over guide wire with stone visible in common hepatic duct; right panel: dredging balloon within bile duct after sphincteroplasty having retrieved the stone.

then inflated with a $4 \mathrm{ml}$ mixture of radio-opaque contrast medium and saline to a pressure of 8 atmospheres and to a maximum diameter of $1 \mathrm{~cm}$ for 60 seconds (Figure). After deflation for 30 seconds the balloon is re-inflated for 60 seconds. A central impression corresponding to the sphincter is visible during balloon inflation (Figure). The dilated papilla permits easy access to dormier basket, 'retrieval' balloon (Figure) or mechanical lithotripsy for extraction of the bile duct stones. Biliary manometry was not performed.

\section{Results}

The bile duct was cleared in 22 of 28 patients (79\%) with sphincterplasty (Table). Stone size ranged from 3 to $20 \mathrm{~mm}$ and the maximum number of stones removed from a single patient was five. Mechanical lithotripsy was used in two patients to extract large stones of $15-20 \mathrm{~mm}$ in diameter. Two patients $(7 \%)$ had a second ERCP (without repeat sphincteroplasty) to achieve duct clearance. In one patient in whom sphincteroplasty was successful, the papilla was located within a large diverticulum. Transient discomfort was noted in about half of the patients during balloon inflation. No procedure related haemorrhage was seen but sphincteroplasty was complicated by pancreatitis in one patient (4\%). This episode was associated with a maximum serum amylase of $6500 \mathrm{IU} / 1$ and resolved within 24 hours after intravenous fluids and analgesia.

Sphincteroplasty alone failed to clear the bile duct in six of 28 patients (21\%) (Table). In four patients (14\%), papillotomy was also required to extract stones up to $20 \mathrm{~mm}$ in diameter after unsuccessful basket retrieval with sphincteroplasty alone. As regards the two remaining patients $(7 \%)$ in whom sphincterplasty failed to clear the bile duct, a biliary prosthesis was inserted with satisfactory drainage. A large papillotomy was considered hazardous in these two patients who were elderly and debilitated with large bile duct stones in whom stone extraction was technically difficult despite a sphincteroplasty. Insertion of an endoprosthesis is now an accepted method of establishing biliary drainage in such patients. ${ }^{8}$

Median follow up of 16 weeks (range 2-44) for all 28 patients did not show any adverse effects associated with sphincteroplasty.

\section{Discussion}

Bile duct stones are reported to occur in about $15-18 \%$ of patients with gall bladder stones. ${ }^{1}$ In the absence of an effective method to clear the bile duct during laparoscopic cholecystectomy, the demand for ERCP will probably increase. ${ }^{12}$ Papillotomy, however, may be associated with complications. ${ }^{34}$ Apart from these immediate complications, concern regarding the longterm sequelae of papillotomy in young patients stimulated our interest in an alternative approach. Our results show that when balloon sphincteroplasty was used, papillotomy was unnecessary to facilitate bile duct clearance in most patients (79\%). Stones were removed by dormier basket or balloon in most patients while mechanical lithotripsy was required for a few with large stones. It is notable that this series included a patient with the papilla located within a large diverticulum, a situation associated with a higher risk of perforation if papillotomy is performed. The procedure is not difficult to perform and requires less skill than papillotomy. In particular, no papillary haemorrhage was seen. There were no complications apart from a single case of pancreatitis, which settled on conservative management within 24 hours. To date, no longer term adverse effects have been reported in any of the patients. The effect of sphincteroplasty on papillary function has yet to be determined.

What are the limitations of sphincteroplasty? We decided on clinical grounds not to use this technique in four patients with stones more than $2 \mathrm{~cm}$ in size to avoid the prospect of impaction during basket retrieval. While large pigment 'soft' stones $(>2 \mathrm{~cm})$ may be crushed and subsequently removed after sphincteroplasty alone, the possibility of impaction with 'hard' stones with the attendant possible requirement for surgery must be borne in mind. Sphincteroplasty alone was unsuccessful in bile duct clearance in six patients (22\%) in our series and this related primarily to technical difficulties in retrieving stones $15-20 \mathrm{~mm}$ in size.

The results show that balloon sphincteroplasty (with or without lithotripsy) is a safe and effective technique that significantly reduces the need for papillotomy in the management of bile duct stones. Although the longterm effect on papillary function is unclear, more widespread application of this sphincter preservation technique including manometric evaluation is warranted to confirm our preliminary encouraging results.

Preliminary data presented to British Society of Gastroenterology Meeting in September 1992 and published in abstract form in Gut 1992; 33 (suppl 2): S33. 
1 Cotton PB, Ballie J, Pappas TN, Meyers WS. Laparoscopic cholecystectomy and the biliary endoscopist. Gastrointest Endosc 1991; 37: 94-7.

2 Cronin KJ, Kerin MJ, Williams NN, Crowe J, Mac Mathuna P Lennon G, et al. Endoscopic management of common duct stones with laparoscopic cholecystectomy. Ir F Med Sci 1991 160: $265-7$.

3 Vaira D, D'anna L, Ainley C, et al. Endoscopic sphincterotomy in a 1000 consecutive patients. Lancet 1989 ; ii: 431-3.

4 Sherman S, Ruffolo TA, Hawes RH, Lehman GA. Complications of endoscopic sphincterotomy: a prospective series with emphasis on increased risk associated with sphincter of Oddi dysfunction and nondilated bile ducts. Gastroenterology 1991; 101: 1068-75.
5 Groen JN, Lock MT, Lameris JS, Van Blankenstein $M$, Terpstra OT. Removal of bile duct stones by the combination of percutaneous balloon dilatation and extracorporeal shockwave lithotripsy. Gastroenterology 1989; 97: 202-6.

6 Staritz M, Ewe K, Meyer zum Buschenfelde KH. Endoscopic papillary dilatation for the treatment of common bile duct stones and papillary stenosis. Endoscopy 1983; 15: 197-8.

Koserak RA. Balloon dilation of the sphincter of Oddi. Endoscopy 1988; 20: 207-10.

8 Peters R, Mac Mathuna P, Lombard M, Karani J, Westaby D. Management of common bile duct stones with a biliary endoprosthesis. Report on 40 cases. Gut 1992; 33: 1412-5. 